\title{
Reanimating the paralyzed face
}

\author{
Kofi Boahene
}

Address: Department of Otolaryngology Head and Neck Surgery, Johns Hopkins Institute of Medicine, 601 N. Caroline Street, Baltimore, MD 21287, USA

Email: dboahen1@jhmi.edu

Fl000Prime Reports 2013, 5:49 (doi:10.12703/P5-49)

This is an open-access article distributed under the terms of the Creative Commons Attribution-Non Commercial License (http://creativecommons.org/licenses/by-nc/3.0/legalcode), which permits unrestricted use, distribution, and reproduction in any medium, provided the original work is properly cited. You may not use this work for commercial purposes.

The electronic version of this article is the complete one and can be found at: http://fl000.com/prime/reports/m/5/49

\begin{abstract}
Facial animation is an essential part of human communication and one of the main means of expressing emotions, indexing our physiologic state and providing nonverbal cues. The loss of this important human quality due to facial paralysis can be devastating and is often associated with depression, social isolation and poor quality of life. Interruption of the neuromuscular pathway from the facial motor cortex to the facial muscles is the common causative factor in facial paralysis resulting from various etiologies. Restoring tone, symmetry and movement to the paralyzed face requires timely nerve grafting intervention in cases of reversible paralysis and the transfer of functional muscle units in irreversible paralysis. We review recent advances in these techniques.
\end{abstract}

\section{Introduction}

Facial animation is an essential part of human communication and one of the main means of expressing emotions, indexing our physiologic state and providing non-verbal cues. The loss of this important human quality due to facial paralysis can be devastating and is often associated with depression, social isolation and poor quality of life. Facial paralysis significantly impairs eyelid closure, nasal breathing, lip competence and speech. The goals of facial reanimation include the correction of these impaired functions and restoration of facial symmetry at rest and in expression. The face can become paralyzed as a result of developmental abnormalities, early childhood infection, vascular compression of the facial nerve, inflammatory processes, primary and secondary facial neoplasms, iatrogenic facial nerve injuries and trauma. Interruption of the neuromuscular pathway from the facial motor cortex to the facial muscles is a common cause of facial paralysis, resulting from various etiologies. Reanimation of the paralyzed face requires restoration of the interrupted nerve-muscle network, either by nerve repair or muscle substitution.

\section{Evaluating the face for reanimation}

When assessing a patient for a possible facial reanimation procedure, it is first important to determine if the muscle paralysis can be reversed. It is also essential to determine the cause of the paralysis, duration, and the functional deficits that are present. Establishing the cause of the facial paralysis gives essential information about the prognosis and expected course of the paralysis. A thorough history is usually adequate to determine the cause of facial paralysis. When necessary, a high resolution magnetic resonance image (MRI) or computerized tomography (CT) scan may be obtained to evaluate the intracranial and extracranial course of the facial nerve and to evaluate the patient for skeletal and soft-tissue abnormalities. Even after a thorough history check and high-resolution imaging studies, the cause of a facial paralysis may remain elusive and a diagnostic surgical exploration to rule out occult neoplasms may be necessary [1]. An accurate assessment of the functional deficits is important in selecting the appropriate reanimation technique and helps prioritize the order of intervention. The various options available for reanimating the face should be explained to patients. A holistic 
approach to facial reanimation with attention to both the paralyzed and non-paralyzed sides of the face yields more satisfying results [2]. This approach gives patients hope and allows them to become an active participant in their rehabilitation.

\section{Is the paralysis reversible or irreversible?}

The potential for reversing facial paralysis depends on the status of the facial muscles. Reversibly paralyzed facial muscles have physiologically viable fibers with intact nerve motor units that will respond to ingrowing axons. Innervating such muscles restores tone and movement to the face. On the contrary, atrophic and fibrotic facial muscles are irreversibly paralyzed and will not respond to reinnervation because of several mechanical and physiologic barriers to incoming axons. These include deteriorated intramuscular nerve sheath and loss of supportive satellite cells [3]. To restore tone and movement in irreversible facial paralysis, a functional muscle is needed to replace the damaged facial muscles. The length of time a facial muscle is denervated is a rough predictor of the reversibility of the facial paralysis. Facial muscles completely denervated for less than 1 year respond to nerve grafting. The response to reinnervation after a year of paralysis diminishes significantly and becomes unpredictable. In cases of incomplete denervation, the facial muscle can remain viable for longer periods and respond to further reinnervation techniques. An electromyogram (EMG) may be performed to determine if a facial muscle paralysis can be reversed. The presence of fibrillation potentials on an EMG suggests an electrically active muscle that may respond to reinnervation.

\section{Timing of intervention}

The duration of denervation is probably the most important factor that determines the ultimate success of any reinnervation procedure. Interrupted facial nerves should be promptly repaired either directly or with an interposition graft. The timing of intervention is not so clear in cases of complete facial paralysis when the facial nerve is anatomically intact. Since spontaneous recovery of the facial paralysis may occur, a period of observation is often recommended. How long this period of observation should be is not clear, but it is a common practice to observe for spontaneous recovery for a period of one year before intervening. However, this approach delays the timely intervention in the subset of patients who ultimately do not recover spontaneously. In a review of 281 patients with an anatomically intact facial nerve following acoustic neuroma resection, Rivas et al. found that the rate of recovery during the first year could be used to predict long-term facial nerve recovery [4]. For patients starting with a House Brackmann grade V or VI facial paralysis, the recovery rate was the most reliable predictor of poor outcome after 1 year. The resulting predictive model using rate of functional improvement as the independent variable was found to anticipate poor outcome before 1 year in more than $50 \%$ of cases with $97 \%$ sensitivity and $97 \%$ specificity. Therefore, when a patient with an anatomically intact facial nerve and a House-Brackmann grade $\mathrm{V}$ or worse facial paralysis shows no improvement of at least one House-Brackmann grade after 6 months of observation, plans for nerve repair can be entertained.

\section{Reanimation of the upper face Brow ptosis}

Paralysis of the frontalis muscle results in ptosis of the brow that may obstruct the peripheral visual field, which is usually more pronounced in elderly patients. In younger patients, aesthetic brow asymmetry is usually more bothersome. By manually elevating the brow, the degree to which brow ptosis contributes to visual field obstruction can be determined. Endoscopic brow suspension for females and younger patients is our main approach to correcting paralytic brow ptosis. In elderly patients, midforehead or a temporal brow lifts have been satisfactory. When suspending the paralyzed forehead we emphasize elevating the temporal portion of the brow. Selective chemodenervation of the contralateral frontalis muscle also helps in achieving forehead and brow symmetry.

\section{Eyelids}

Paralysis of the orbicularis oculi muscle is associated with significant eyelid dysfunction that can lead to keratitis, corneal abrasion and ulceration. Restoring eyelid function and corneal protection is one of the primary focuses of reanimation efforts in facial paralysis. Preoperative assessment of the paralyzed eyelid should systematically document the ability of the patient to voluntarily and involuntarily blink, corneal sensation, globe position (positive vs. negative vector globe), medial and lateral canthal laxity, position of lower lacrimal punctum, tearing and the degree of lower eyelid retraction. We address the upper and lower eyelids separately with either static procedures, regional muscle transfer or nerve grafting procedures based on patient age, degree of lagophthalmos, ectropion and reversibility of the paralysis. In reversible paralysis, nerve grafting techniques are preferred to static procedures since they yield long-term eyelid tone and orbicularis oculi muscle preservation. Even when distal facial nerve fibers to the orbicularis oculi muscle cannot be identified, muscle tone can be restored by directly neurotizing the muscle with a nerve graft. Static eyelid procedures are used as adjuncts to nerve grafting techniques and for irreversible paralysis. 


\section{Upper eyelid loading}

Loading the upper eyelid is a static means of improving or correcting the lagophtalmos of blink and the scleral show that is characteristic of complete facial paralysis. Upper eyelid loading is partly recommended for its effect on blink efficiency [5]. Complete and efficient blinking distributes protective tear film over the ocular surface, which is essential for corneal health. Upper eyelid loading is more effective when combined with blink efficiency exercises. Patients are encouraged to consciously practice voluntary blinking with a focus on reproducing the actions of an effective blink. Gold and platinum weights are the most commonly used eyelid implants but several authors have published their experience using cartilage grafts as upper eyelid loads. A major draw back of eyelid loading is the mechanical ptosis that results from the weight leading to palpebral asymmetry and visual field obstruction. Weights between 0.8-1.0 g induce less ptosis and can be very effective when combined with frequent voluntary blink efficiency exercises (Fig. 1). Upper eyelid loading is usually not effective in patients with relative proptotic and negative vector eyes.

\section{Lower eyelid spacer graft}

Paralysis of the orbicularis oculi muscle results in the loss of lower eyelid tone and vertical height. A middle lamella spacer graft placed deep to the orbicularis oculi muscle can partially correct the loss of tone and vertical height by adding volume and a layer of reactive fibrosis that introduces some support to the paralyzed lid [6]. The ideal spacer graft should be flexible and not too stiff. We have found the Dura regeneration matrix DuraGen (Integra LifeSciences Corporation) a useful allograft for

Figure I. Upper eyelid loading with platinum chain implant
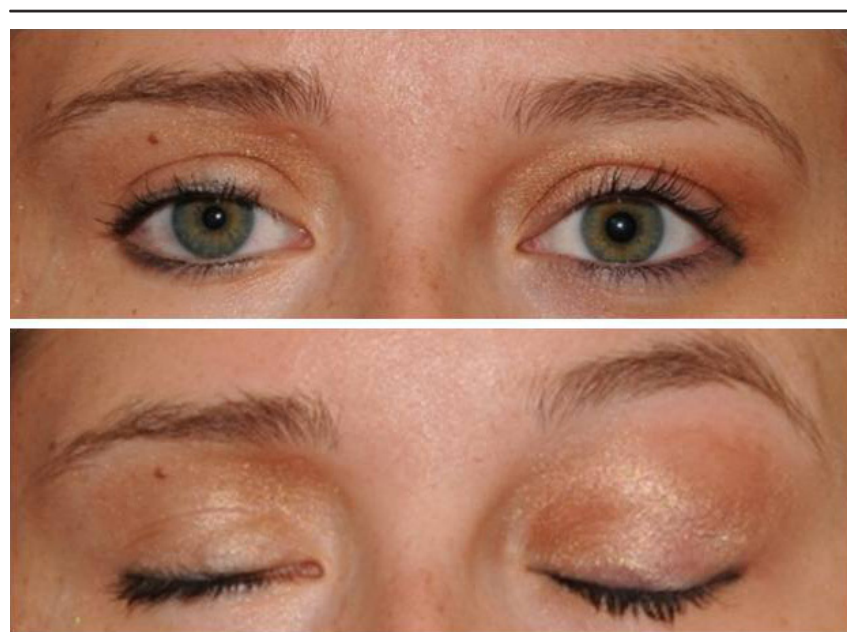

Upper eyelid loading with platinum chain implant to improve blink reflex and eyelid closure in facial paralysis.
Figure 2. Spacer graft placed in the lower eyelid

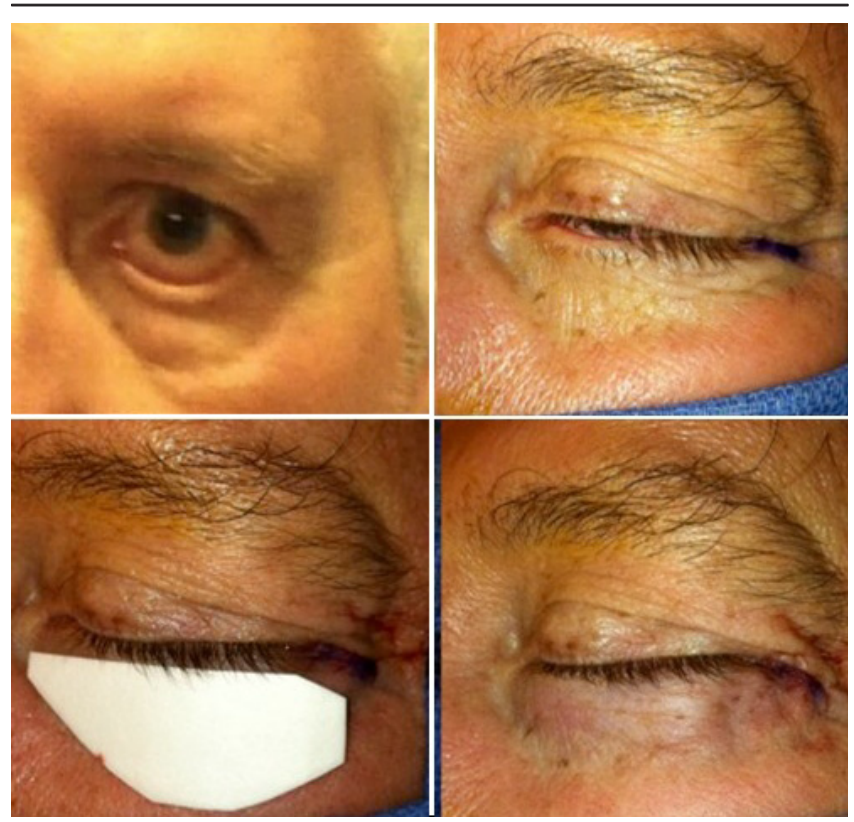

Spacer graft placed in the lower eyelid vertically elevates the paralyzed eyelid to improved cornea protection. The spacer graft material is a collagen regeneration matrix DuraGen® (Integra LifeSciences Corporation).

use as a spacer graft. A lower eyelid spacer graft can be placed through a conjunctival incision or an external lateral canthal incision using a minimally invasive technique [5]. A space is dissected superficial to the orbital septum extending from the eyelid margin to the orbital rim. The lower eyelid retractor, the capsulopalpebral ligament, is bluntly released. The spacer graft is trimmed to take the shape of the lower eyelid but is slightly oversized in its vertical dimension to match the distance from the lower limbus to the orbital rim. The graft is placed in the dissected space with the aid of forceps and smoothed out to eliminate bulges and irregularities. Patients should be cautioned about lid swelling, which resolves over several weeks (Fig. 2).

\section{Lower eyelid suspension}

The retracted lower eyelid can also be vertically suspended with fascia slings placed along the margin of the lower eyelid and anchored medially and laterally. Fascia may be obtained from the fascia lata or from the deep temporal fascia. The sling is placed deep to the orbicularis oculi muscle and secured to periosteum lateral and superior to the attachment points of the canthal ligaments.

\section{Smile restoration: reanimation of the paralyzed midface}

A major focus of facial reanimation procedures is the restoration of midfacial symmetry at rest and oral 
commissure excursion when smiling. In reversible paralysis, restoring neural input into the denervated facial muscles using various nerve grafting and nerve transposition techniques should always be the first option. In irreversible paralysis, the transfer of functional muscle units to the face is necessary to restore facial movement.

\section{Nerve grafting techniques}

Nerve grafts are performed to re-establish electrical continuity between the paralyzed facial muscle and a motor nucleus at the level of the brain stem. When feasible, the denervated facial muscle should be reconnected with the ipsilateral facial nucleus either by direct nerve repair or with an interposition nerve graft. When the ipsilateral proximal facial nerve stump cannot be surgically recruited or is functionally unavailable, alternative donor nerves are considered. The contralateral facial nerve, the masseter nerve and the hypoglossal nerve are the most commonly used donor nerves for facial reanimation.

Direct nerve repairs and interposition nerve grafts are best performed soon after the facial nerve interruption when the proximal and distal ends of the facial nerve can be easily identified. Interposition nerve grafts carefully sutured between the distal and proximal ends of the disrupted facial nerve act as a conduit for axonal regrowth. The sural, great auricular and the ante brachial cutaneous nerves are easily obtained for grafting with minimal morbidity. The sural nerve is a popular choice because of the long length that can be harvested. There is some evidence that motor nerve conduits may be better than sensory nerve conduits $[7,8]$. However, harvesting a motor nerve as conduit may result in an unacceptable morbidity. Motor nerve allografts are now commercially available for use for gaps up to $5 \mathrm{~cm}$. The motor nerve to the vastus lateralis muscle has also been used as a nerve graft on the rare occasion where facial reconstruction required the simultaneous need for a vastus lateralis thigh free flap and facial nerve [9]. A common clinical scenario when interposition nerve graft is needed is following radical parotidectomy for malignancy. In such cases, the proximal facial nerve stump is identified at the stylomastoid foramen or exposed by drilling out the mastoid. The distal nerve stumps to the facial muscles are identified with a nerve stimulator. The donor nerve graft is sutured to the proximal facial nerve stump. The distal end of the donor nerve is dissected into fascicles allowing multiple nerve anastomoses (Fig. 3). Priority is given to the nerves to the orbicularis oculi muscle and branches innervating the upper lip elevators for smile restoration. The interposition donor nerve graft is oriented in a manner that minimizes escape of regenerating axons, channeling them towards the distal facial nerve stump.
Figure 3. Interposition graft with split sural nerve

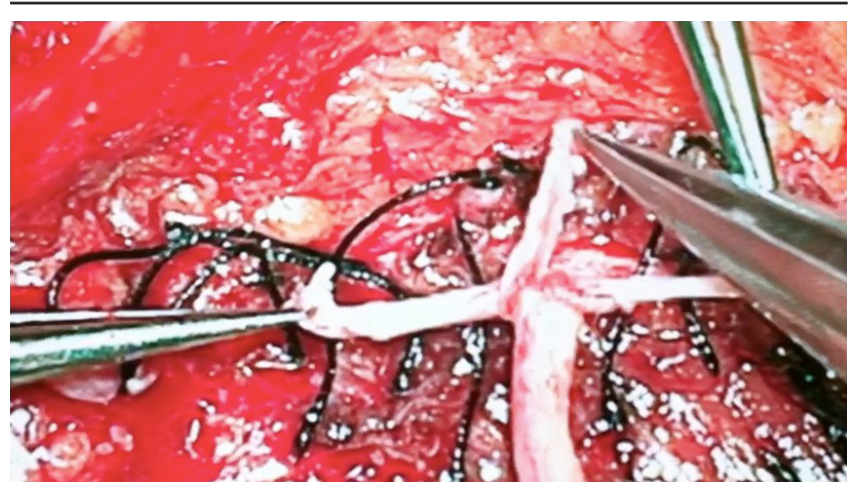

Harvested sural nerve is split into nerve fascial, which are coapted to the severed distal facial nerve branches (tagged with black sutures). The main stump of the sural nerve is coapted to the proximal facial nerve stump in the mastoid.

Nerve coaptation can be performed using suture techniques, tissue glue, nerve conduits, nerve wraps or a combination of these. Stems cells engineered to secrete neurogenic growth factors into the microenvironment of the repaired nerves have been promising in experimental models but are not clinically proven [10].

When the proximal facial nerve stump is not available, alternative motor nerves are required for axonal regeneration. Branches of the contra-lateral facial nerve may be recruited for cross-facial grafting. Successful cross-facial nerve grafting allows for coordinated and volitional mimetic movement by rerouting facial nerve axons from the unaffected side to the paralyzed side through a donor nerve conduit. When successful, cross-facial nerve grafting has the potential of producing spontaneous movement that parallels that of the normal side.

The masseteric nerve is a valuable source for new neural input in the management of facial paralysis. In patients with reversible facial paralysis, the proximity of the masseteric nerve to the facial nerve provides the potential for a tension-free anastomosis to the main trunk or peripheral branches of the facial nerve without the need for an interposition graft. The masseter nerve is a small but dense nerve with over 2700 myelinated motor axons, which can be reliably identified in a minimally disruptive manner using the subzygomatic triangle as an anatomic landmark [11]. The inferior border of the zygomatic arch, a vertical line through the anterior margin of the temporomandibular joint, and the frontal branch of the facial nerve define the subzygomatic triangle (Fig. 4). A line bisecting the angle between the temporomandibular joint line and the zygomatic arch corresponds to the course of the masseter nerve. Through a preauricular 
Figure 4. The subzygomatic triangle
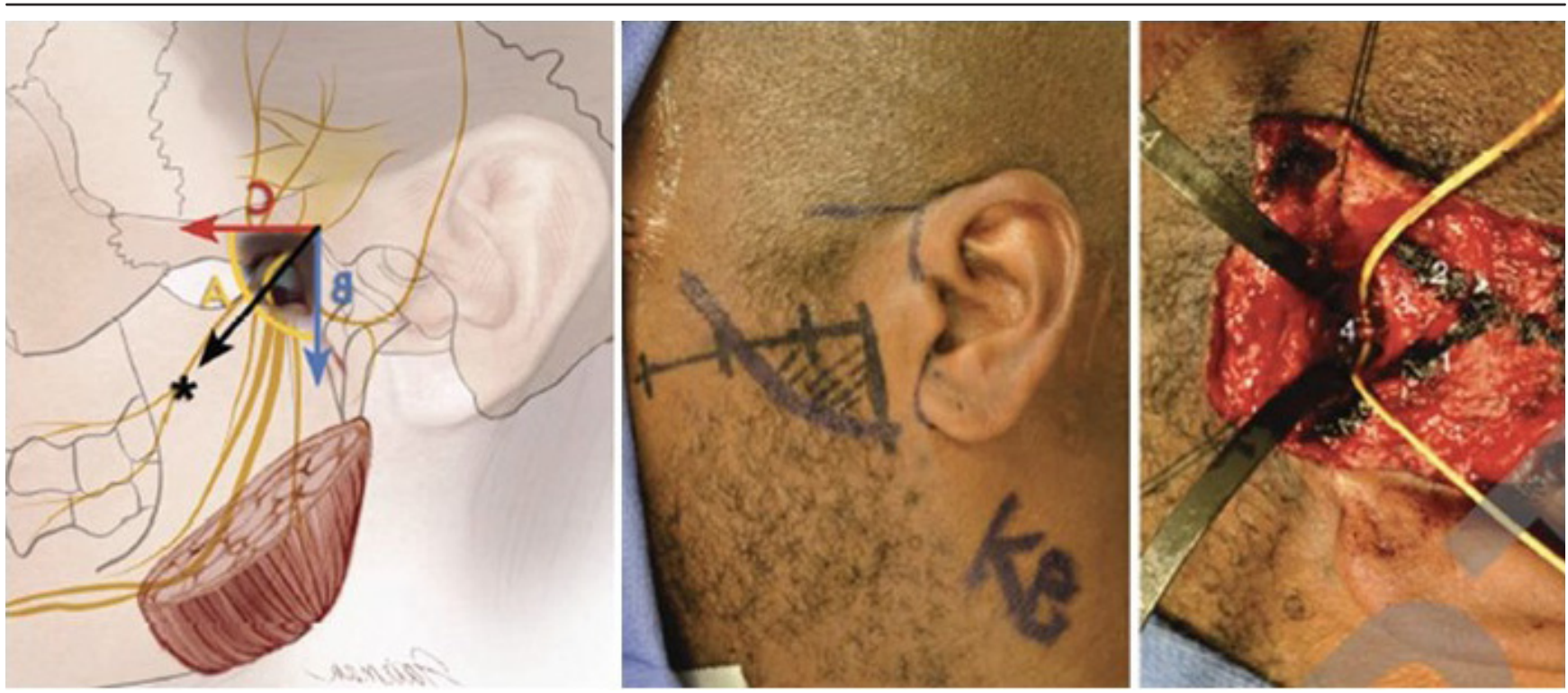

The masseter nerve can be reliably identified within the subzygomatic triangle (shaded area, middle panel) defined by the inferior border of the zygomatic arch (C), anterior border of the temporomadibular joint (B) and the frontal branch of the facial nerve. The masseter nerve courses along a line bisecting the suzygomatic triangle (asterisk).

Figure 5. Results 4 months after masseter nerve transfer

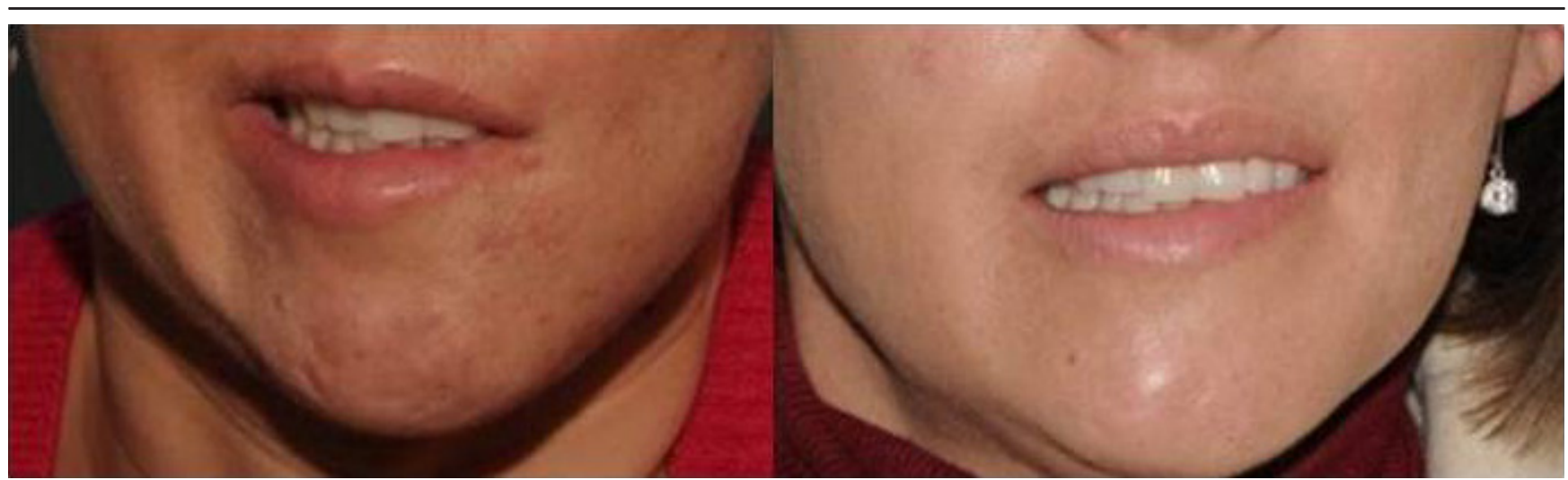

Results 4 months after masseter nerve transfer to the main branch of facial nerve. Notice improved excursion of left oral commissure and dental show when smiling.

incision, the superficial musculoaponeurotic system flap is elevated off the parotid capsule and blunt dissection is used to identify and preserve the frontal branch of the facial nerve. Identifying the frontal branch allows its safe mobilization and retraction to expose the subzygomatic triangle. Deeper dissection exposes the masseter muscle. Within the small area within the subzygomatic triangle, the masseter nerve can be identified, aided by the nerve stimulator. Once identified, the nerve is traced inferiorly for length. Tracing and mobilizing $3 \mathrm{~cm}$ of the masseter nerve allows direct coaptation to the main facial nerve trunk or selected target branches. Tone and movement following masseter to facial nerve transfers are usually seen 4 to 6 months following surgery (Fig. 5). Patients are then directed to practice smiling while biting down to reveal a full smile showing their teeth and with controlled oral commissure excursion.

The hypoglossal nerve has long been used as a source of new axons to reinnervate the paralyzed face. Complete 
transfer of the hypoglossal nerve to the facial nerve provides a high density of axons to the facial muscle that can result in undesirable hypercontracture and to avoid this, partial use of the hypoglossal nerve is often recommended. Unlike the masseter nerve, the location of the hypoglossal nerve in the neck prevents direct coaptation to the facial nerve without a jump graft or splitting and transposing the hypoglossal nerve. The author's preferred method for hypoglossal to facial nerve transfer is the complete exposure and mobilization of the vertical segment of facial nerve from the mastoid and transposing it to the neck for an end-toside coaptation to the hypoglossal nerve. With the vertical segment of the facial nerve skeletonized, the facial nerve is totally mobilized from the stylomastoid foramen and dissected free up to the pes anserinus. The mobilized facial nerve is transposed into the neck. Neurotomy through about 30 to $40 \%$ of the hypoglossal nerve recruits enough axons for tone and movement of the face without hypercontraction. In addition, innervation to the hemi-tongue is preserved. With the hypoglossal to facial nerve transposition, symmetry of the oral commissure and midface is restored. To smile, the patient subtly moves the tongue to contract the zygomaticus major muscle (Fig. 6).

Unlike the cross-facial nerve, movement from the masseter or hypoglossal nerve transposition requires conscious effort and is not readily spontaneous. Persistent practice and adaptation of the restored movement to social settings is necessary to restore some degree of spontaneity over time. An attractive option of nerve transposition is to combine the advantages of the crossfacial nerve graft and the masseter or hypoglossal nerves in dual innervation. In this case, the reliable masseter or hypoglossal nerves produce tone and the cross-facial nerve graft provides coordinated spontaneous movement with the contralateral side.

\section{Functional muscle transfer}

When the facial muscles are irreversibly injured or congenitally absent, transfer of functional muscle units is necessary to restore facial tone, symmetry and movement. Functional muscle units can be transferred as a pedicled muscle tendon unit or as a free functional muscle that requires microsurgical revascularization and nerve anastomosis. In muscle tendon unit transfer, the tendon of a functioning muscle is detached, mobilized, and reinserted into another tendon or bone to substitute for the action of a nonfunctioning muscle. The motor nerve and blood supply of the transferred muscle tendon unit remains intact. The temporalis muscle is commonly transferred as a muscle tendon unit to restore upper lip symmetry and movement in facial paralysis $[12,13]$. Temporalis muscle tendon unit transfer is an effective means of restoring symmetry, tone and movement to the midface in a single-stage procedure. The temporalis muscle tendon unit has been described by Labbe, as a lengthening myoplasty [13]. In the temporalis lengthening myoplasty, the temporalis muscle is exposed through a scalp incision and the posterior third of the muscle released and elevated from its periosteal attachment. The zygomatic arch is then removed to expose the coronoid process and the temporalis tendon. The coronoid process with the attached temporalis tendon is osteotomized. The released temporalis muscle tendon unit is lengthened towards the lip commissure and secured. When releasing the temporalis muscle, care is taken to avoid injury to the neurovascular supply deep to the muscle. We have described an alternative minimally invasive approach to the temporalis muscle tendon unit procedure that does not require an external approach or

Figure 6. Results after transposing the vertical segment of the facial nerve to the hypoglossal nerve with end-to-side coaptation

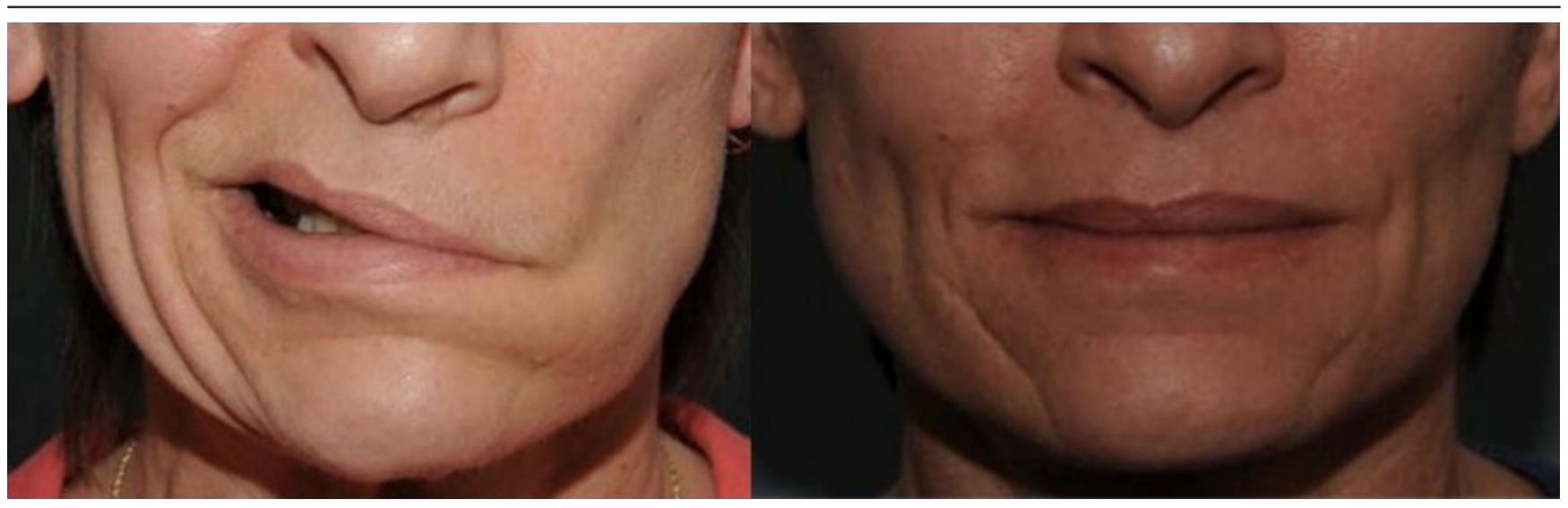

Neurotomy through the hypoglossal nerve is made distal to the ansa cervicalis take off and through 30 to $40 \%$ of the hypoglossal nerve. 
zygomatic arch removal but rather approaches the coronoid process through the buccal space [12]. With this approach, a 2 to $3 \mathrm{~cm}$ incision is made along the nasolabial fold or intraoral through the buccal mucosa. Dissection through the buccal fat exposes the anterior margin of the mandibular ramus and the coronoid. The temporalis tendon is then released together with a small piece of the coronoid. The temporalis tendon is then transposed and secured to the lip. Regardless of the technique used to mobilize the temporalis tendon, the movement that is generated depends on the tension at which the muscle tendon unit is inserted on the lip. Understanding the relationship between muscle length, tension and force generation is essential in optimizing the dynamic range, excursion and force generation of a transferred muscle tendon unit [14]. The biomechanical determinant of active muscle force after transfer is the classic Blix curve. The Blix curve depicts the relationship between active force, passive force and muscle length. A muscle's resting length represents the position from which the muscle can generate its greatest force. Setting the temporalis muscle tendon unit near its normal resting length is probably the most important factor in achieving maximal force generation and excursion from a transferred muscle tendon unit. Using electrical stimulation of the temporalis muscle, intraoperative excursion of the temporalis muscle tendon unit after its release can be determined while varying the traction tension on the tendon [15]. The traction tension at which maximum amplitude of excursion of the temporalis muscle tendon unit is recorded is selected as the ideal tension at which the tendon is inserted to maximize post-operative excursion.

Patients who have undergone the temporalis tendon transfer procedure are usually satisfied with the improved symmetry of the lips that results (Fig. 7). The degree of dynamic movement they achieve depends significantly on their ability to learn to deploy the temporalis muscle when they smile. Active physical therapy to acquire the temporal smile should be strongly encouraged and possibly started prior to the surgery to fully engage patients when they are not sore and swollen from surgery.

Functional muscle can be transferred to the paralyzed face as free functional muscle units that require microsurgical revascularization and reinnervation. One major advantage of free functional muscle transfer is the ability to couple movement of the paralyzed face with that of the unparalyzed contralateral face by using cross-facial nerve grafts. Several donor muscles have been described including the gracilis, latissimus, pectoralis minor and serratus muscles. Gracilis muscle transfer is the most widely described free functional muscle transfer for facial reanimation (Fig. 8). The gracillis muscle is an adductor muscle of the thigh and is innervated by the obturator nerve (L3, L4). The dominant arterial supply to the muscle is a branch of the medial circumflex artery or the deep femoral artery. An arterial and venous pedicle length of $5 \mathrm{~cm}$ to $6 \mathrm{~cm}$ can be commonly harvested. The obturator

Figure 7. Improved symmetry of the lips after temporalis tendon transfer

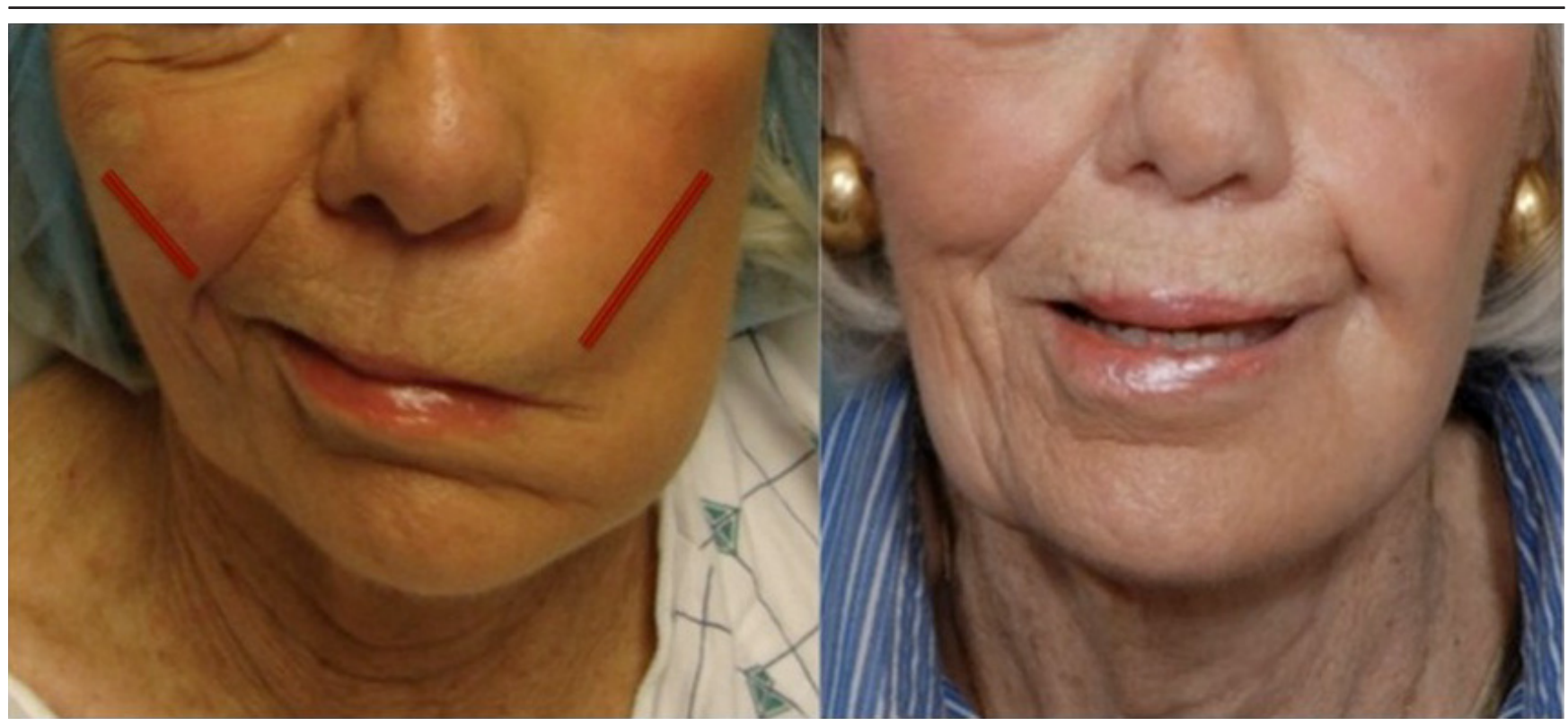

Results after a minimally invasive approach to the temporalis tendon transfer for facial reanimation. 
Figure 8. Gracilis free functional muscle transfer

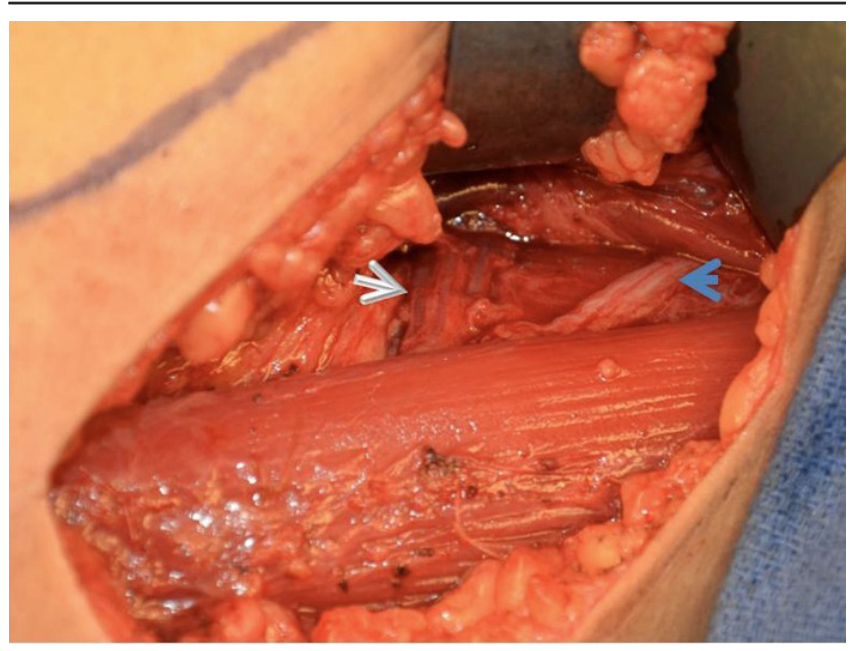

Gracilis free functional muscle transfer for reanimation of the irreversibly paralyzed facial muscle. Vascular pedicle (white arrow head) and obturator nerve (blue arrow head) to gracilis muscle.

nerve commonly has four fascicles, a larger one that supplies the anterior portion of the gracilis muscle and three smaller ones. As the gracilis muscle has a muscle cross sectional area larger than the upper lip elevators it is replacing, a split gracilis muscle is often harvested for facial reanimation. Using selective nerve stimulation, the segment of the muscle that is innervated by the dominant nerve fascicle is harvested. As with the temporalis muscle tendon unit, consideration of the biomechanical principles of muscle transfer is essential to provide an optimal outcome in free functional gracilis transfer. Setting the transferred gracilis at the length and tension close to its passive length and tension in the leg yields the best result. Suture markers placed on the gracilis at measured intervals help with reinserting the transplanted muscle close to its passive length. Methods that can help determine sarcomere length during the procedure will be more accurate in maintaining muscle length and tension. Additional points of technical finesse help with the overall result. First, to maintain facial symmetry, muscle bulk should be minimized. Second, the vector of pull should closely mimic that of the zygomaticus major. Third, a generous fat layer should be preserved on the cheek skin flap to maintain a glide plane and avoid scarring of the transplanted muscle to the overlying skin. Fourth, the muscle should be inserted close to the lip margin for better lip excursion and teeth exposure during smile. Commonly used donor nerve sources for reinnervating the transplanted free functional muscles include the cross-facial nerve graft, masseter nerve and the hypoglossal nerve. The cross-facial nerve has the potential for coupling movement of the reanimated face to the normal contralateral face for a more spontaneous movement. Cross-facial nerve grafts are commonly placed during the first stage of the reconstruction and the functional muscle transfer performed as a second stage procedure 6 to 9 months later. Adequate axons are needed to traverse the cross-facial graft to allow adequate motor input into the transplanted muscle. As such, a sizeable donor facial branch from the normal side should be selected. The masseter nerve is increasingly being used to power free functional muscle transplants [16], as it is readily available in the facial region and allows a singlestage procedure. The fascicular arrangement of the obturator nerve allows for dual innervation of the free functional muscle transplant using a combination of a cross-facial and masseter nerve, either as a single-stage procedure or a two-stage procedure 6 to 9 months after a first-stage cross-facial nerve graft [17].

\section{Assessment and reanimation of the lower face}

The lower lip is important for maintaining lip competence and in the generation of plosive sounds during speech. The depressor muscles of the lower lip contribute in the production of a full smile that reveals both the upper and lower lip. Isolated injury to the marginal branch of the facial nerve is common after neck dissections and parotidectomy and results in a distorted smile. Patients with lip muscle atrophy and lip incompetence as a result of long-standing paralysis may benefit from lip augmentation. Augmentation of the atrophic lip can be achieved with hyaluronic acid filler, fat or rolled fascia. Lower lip symmetry is commonly addressed with chemodenervation of the normal active depressor labia muscle. Once patients express satisfaction with the effect of the temporary denervation of the depressor labia, a more permanent result can be achieved by selective neurectomy of the marginal branch or a segmental resection of the depressor labia muscle. Some patients complain of biting their lower lips because the paralyzed lower lip rolls in, and a muscle transfer procedure is more corrective in these patients than destruction of the contralateral depressor labia muscle.

Changes in the neck following facial paralysis are mainly seen in the platysma muscle and may be negligible or severe (Fig. 9). The platsyma muscle extends over the mandible and it continues with the superficial musculoaponeurotic system of the midface. Aberrant reinnervation of the platysma muscle following facial nerve injury often manifests as a hypercontracture of the platysma muscle that produces a tight feeling in the neck. As a depressor of the lower face, hypercontraction of the platysma muscle is a major antagonist to lip elevation and can impair movement of the upper lip when smiling. Resection of the hypercontracting platysma muscle or temporary 
Figure 9. Changes following resection of a segment of the platysma muscle

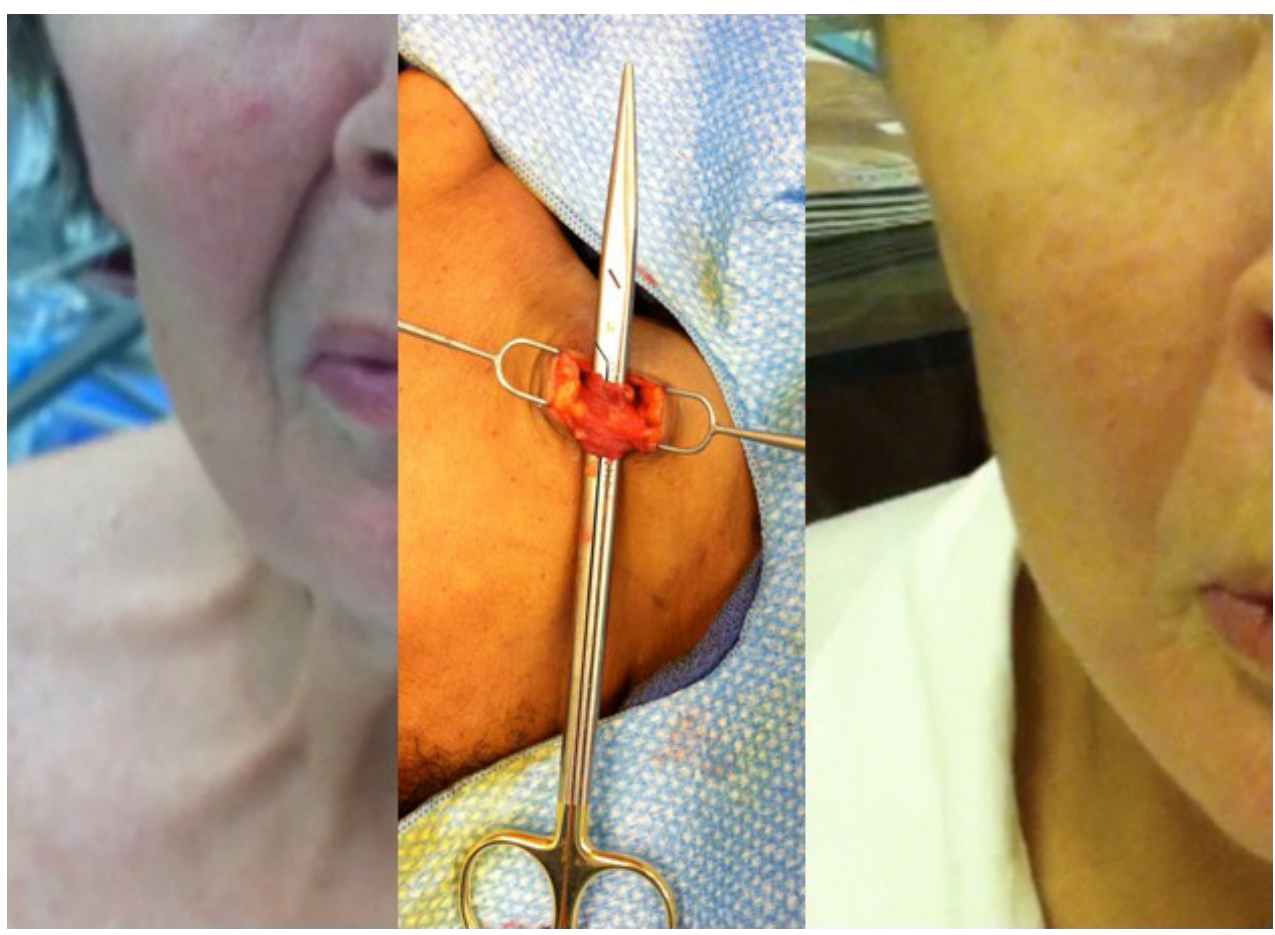

Platysma hypercontacture contributing to facial tightness, synkinesis and restricted movement of the oral commissure (left hand panel). Resection of a segment of the platysma muscle (centre panel) improves the facial tightness and synkinesis and may allow improved excursion of the oral commissure (right hand panel).

chemodenervation relieves the facial tightness and may allow better excursion of the lip elevators [18]. In assessing for platysma resection, patients are asked to smile broadly and the presence and location of tight platysma bands are marked. Through a $2 \mathrm{~cm}$ neck incision, a broad segment of the hypertrophic platysma muscle is resected to interrupt the hyper-functioning muscle. Patients often express immediate relief of neck and facial tightness as well as improvement in their smile. Small subsets of patients complain of a worsening of their smile. This latter subset of patients seem to depend significantly on their platysma muscle for lower lip movement and appear to like the perception of contraction from the hyperactive platysma muscle, even when it produces invisible lip movement. These patients may be identified using a trial of platysma chemodenervation and one can then avoid myectomy of the platysma muscle.

\section{Adjunctive therapy}

Current facial reanimation strategies fall far short of completely restoring the complex expressive movements and functional characteristic of the normal unparalyzed face. This is partly because facial paralysis affects the facial neuromuscular network at multiple levels, including the facial nucleus, facial nerve fibers and the facial muscles. Also, changes that occur in the facial nucleus and the facial motor cortex after prolonged facial paralysis cannot be directly corrected with reanimation surgery. To influence the surgically inaccessible aspects of the facial neuromuscular network, adjunctive therapies are needed. Facial neuromuscular retraining and the selective use of chemodenervation agents are the main nonsurgical interventions commonly recommended for facial paralysis.

Neuromuscular facial re-education was introduced in 1980 in the Netherlands, specifically for patients with facial nerve paralysis, through collaborative work between mime-actors and clinicians. Neuromuscular retraining unlinks undesired motions from desired ones using slow, small-amplitude, desired motions while consciously suppressing the undesired ones. As the undesired activity is suppressed, the range of the primary movement gradually extends, increasing excursion, strength, and motor control. Surface EMG and the use of mirror and video biofeedback helps bring desired movements under conscious control. Once the patients have become comfortable with self-directed facial retraining exercises, selective chemodenervation with 
botulinum toxin injection can add significant benefit. The selective use of botulinum toxin helps uncouple facial muscle groups involved in synkinetic movement, spasm and hypercontraction. Treating both the paralyzed and unparalyzed face produces balance and improves symmetry.

\section{Summary}

Facial expression is a complex activity resulting from a delicate network of facial nerves and muscles. Facial paralysis resulting in the loss of characteristic expressions can be devastating. Restoring tone, symmetry and movement to the paralyzed face requires timely nerve grafting intervention in cases of reversible paralysis and the transfer of functional muscle units in irreversible paralysis. In the case of functional muscle unit transfer, adhering to the biomechanical principles relating muscle tension to excursion is necessary to yield an optimal result. Adjunctive facial retraining therapy and the selective use of chemodenervation agents can complement and enhance the final result of reanimation techniques.

\section{Abbreviations}

$\mathrm{CT}$, computerized tomography; EMG, electromyogram; MRI, magnetic resonance imaging.

\section{Disclosures}

The author declares that he has no disclosures.

\section{References}

I. Boahene K, Olsen KD, Driscoll C, Lewis JE, McDonald TJ: Facial nerve paralysis secondary to occult malignant neoplasms. Otolaryngol Head Neck Surg 2004, 130:459-65.

2. Boahene $K$, Byrne $P$, Schaitkin BM: Facial reanimation: discussion and debate. Facial Plast Surg Clin North Am 2012, 20:383-402.

3. Fu SY, Gordon T: Contributing factors to poor functional recovery after delayed nerve repair: prolonged denervation. J Neurosci 1995, I5:3886-95.

\section{FlOOOPrime
RECOMMENDED}

4. Rivas A, Boahene KD, Bravo HC, Tan M, Tamargo RJ, Francis HW: A model for early prediction of facial nerve recovery after vestibular schwannoma surgery. Otol Neurotol 201 I, 32:826-33.

5. McMonnies CW: Incomplete blinking: exposure keratopathy, lid wiper epitheliopathy, dry eye, refractive surgery, and dry contact lenses. Cont Lens Anterior Eye 2007, 30:37-5I.

\section{FlOOOPrime \\ RECOMMENDED}

6. Chang HS, Lee D, Taban M, Douglas RS, Goldberg RA: “En-glove” lysis of lower eyelid retractors with AlloDerm and dermis-fat grafts in lower eyelid retraction surgery. Ophthal Plast Reconstr Surg 20II, 27:137-4I.

7. Lloyd BE, Luginbuhl RD, Brenner MJ, Rocque BG, Tung $T H$, Myckatyn TM, Hunter DA, Mackinnon SE, Borschel GH: Use of motor nerve material in peripheral nerve repair with conduits. Microsurgery 2007, 27:38-45.

\section{FIOOOPrime}

8. Brooks DN, Weber RV, Chao JD, Rinker BD, Zoldos J, Robichaux MR, Ruggeri SB, Anderson KA, Bonatz EE, Wisotsky SM, Cho MS, Wilson C, Cooper EO, Ingari JV, Safa B, Parrett BM, Buncke GM: Processed nerve allografts for peripheral nerve reconstruction: a multicenter study of utilization and outcomes in sensory, mixed, and motor nerve reconstructions. Microsurgery 2012, 32:1-14.

\section{FlOOOPrime}

9. Revenaugh PC, Knott PD, McBride JM, Fritz MA: Motor Nerve to the Vastus Lateralis. Arch acial Plast Surg 2012.

10. Jesuraj NJ, Santosa KB, Macewan MR, Moore AM, Kasukurthi R, Ray WZ, Flagg ER, Hunter DA, Borschel GH, Johnson PJ, Mackinnon SE, Sakiyama-Elbert SE: Schwann cells seeded in acellular nerve grafts improve functional recovery. Muscle Nerve 2013.

\section{FlOOOPrime}

\section{RECOMMENDED}

II. Collar RM, Byrne PJ, Boahene KD: The Subzygomatic Triangle: Rapid, minimally invasive identification of the masseteric nerve for facial reanimation. Plast Reconstr Surg 2013, 132:183-8.

12. Boahene KD, Farrag TY, Ishii L, Byrne PJ: Minimally invasive temporalis tendon transposition. Arch Facial Plast Surg 201I, 13:8-13.

\section{FlOOOPrime}

RECOMMENDED

13. Labbé $D$, Huault M: Lengthening temporalis myoplasty and lip reanimation. Plast Reconstr Surg 2000, I05:1289-97.

\section{FlOOOPrime
RECOMMENDED}

14. Fridén J, Lieber RL: Mechanical considerations in the design of surgical reconstructive procedures. J Biomech 2002, 35: 1039-1045.

\section{FlOOOPrime
RECOMMENDED}

15. Boahene KD: Principles and biomechanics of muscle tendon unit transfer: Application in temporalis muscle tendon transposition for smile improvement in facial paralysis. Laryngoscope 2013, I 23:350-5.

16. Hontanilla B, Marre D, Cabello A: Facial reanimation with gracilis muscle transfer neurotized to cross-facial nerve graft versus masseteric nerve: A comparative study using the FACIAL CLIMA Evaluating System. Plast Reconstr Surg 2013, I 3 I: | 24 |-52. FlOOOPrime RECOMMENDED

17. Biglioli F, Bayoudh W, Colombo V, Pedrazzoli M, Rabbiosi D: Double innervation (facial/masseter) on the gracilis flap, in the middle face reanimation in the management of facial paralysis: A new concept. Ann Chir Plast Esthet 2013, 58:89-95.

\section{FlOOOPrime}

\section{RECOMMENDED}

18. Henstrom DK, Malo JS, Cheney ML, Hadlock TA: Platysmectomy: an effective intervention for facial synkinesis and hypertonicity. Arch Facial Plast Surg 201 I, I3:239-43.

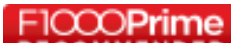

RECOMMENDED 